\title{
Notes regarding the Modeling of the Angle of Attack
}

\author{
Laurentiu M oraru, ${ }^{1,}$ \\ ${ }^{1}$ The University Politehnica of B ucharest, A erospace Sciences Department, R omania
}

\begin{abstract}
Numerical integration has become routine for many decades and so has become the numerical integration of the aircraft's equation of motion. M any numerical algorithms have been used in flight dynamics and the applications of the basic numerical methods to flight simulation have been included in textbooks for a long time. However, many design and/or optimization algorithms rely on analyzing large amounts of simulated data, so analytical algorithms that can provide expedite estimations of the fast varying parameters have been revaluated. The current paper discusses approximate analytical solutions for the angle of attack. Two types of such solutions are discussed. The first model considered originates in the classically linearized equations of motion. The second model discussed was obtained by simplifying the nonlinear equations of motion. The two models are compared against numerical results, provided by classical numerical integration algorithms.
\end{abstract}

\section{Introduction}

A pproximate analytical solutions were widely used in engineering, including aerospace engineering, before computers became cheap and readily available. Analytical solutions for flight dynamics that were developed before the "computer revolutions relied on series solutions, special functions (sometimes called trocket functions $\int$ ) and eventually complex variables. These analytical solutions were often written in terms of combinations of variables (for example, function of Tarc-lengthJ or various parameters including the space flown along the trajectory), they were many pages long and not easy to implement. D etails can be fond in the literature from the fifth to the eighth decades of the twentieth century, for example Refs. [1-6]. These analytical models, although quite long (often several pages long), allowed progress to be made; however, many of them have been continuously phased out by numerical methods.

Numerical methods have become routine for many decades and so has become the numerical integration of the aircraft's equation of motion. M any numerical al gorithms have been used in flight dynamics and the applications of the basic numerical methods to flight simulation have been included in textbooks for a long time. However, many modern design and/or optimization algorithms rely on analyzing large amounts of computer generated data, often pushing computers and algorithms to the limits. Consequently analytical

${ }^{*}$ Corresponding author: laurentiu.moraru@ gmail.com 
algorithms that can provide expedite estimations of the fast varying parameters have been revaluated, refs. [7] and [8].

The current paper discusses approximate analytical solutions for the angle of attack. Two types of such solutions are discussed. The first model considered originates in the classically linearized equations of motion. The second model discussed was obtained by simplifying the nonlinear equations of motion using an order magnitude analysis. The two models are compared against numerical results, provided by classical numerical integration algorithms.

\section{Models for the angle of attack}

The current paper discusses models for the angle of attack for airplanes and nonspinning rockets in symmetric flight. Analytical solutions are sought, hence assumptions are made to enable approximate solutions to be obtained. The analytical solutions discussed below were obtained for the cases when the variation of mass is slow enough so the inertial properties can be considered constant for short time intervals.

\subsection{Nonlinear model}

The equations of motion for symmetric flight are presented in virtually all the college level textbooks in the field; neglecting the variation of altitude over time intervals comparable to the period of the fast modes, the equations that describe the longitudinal dynamics, hence the angle of attack are.

$$
\begin{aligned}
& m \frac{d V}{d t}=T \cos \alpha-\bar{q} S C_{D}-m g \sin \gamma \\
& m V \frac{d \gamma}{d t}=T \sin \alpha+\bar{q} S\left(C_{L 0}+C_{L \alpha} \alpha+C_{L \delta e} \delta e+C_{L q} q+C_{L \alpha} . \dot{\alpha}\right)-m g \sin \gamma \\
& J_{y} \frac{d q}{d t}=\bar{q} S c\left(C_{m 0}+C_{m \alpha} \alpha+C_{m \delta e} \delta e+C_{m q} q+C_{m \dot{\alpha}} . \dot{\alpha}\right) \\
& \frac{d \alpha}{d t}=q-\frac{d \gamma}{d t}
\end{aligned}
$$

\subsection{The angle of attack small perturbations model}

Standard small perturbation linearization techniques led to the classical linear system, which is written here as

$$
\begin{aligned}
& \frac{d}{d t} \delta V=A_{V} \delta V+A_{\gamma} \delta \gamma+A_{\alpha} \delta \alpha+A_{q} \delta q+A_{\delta e} \delta e \\
& \frac{d}{d t} \delta \gamma=B_{V} \delta V+B_{\gamma} \delta \gamma+B_{\alpha} \delta \alpha+B_{q} \delta q+B_{\alpha} \delta \dot{\alpha}+B_{\delta e} \delta e \\
& \frac{d}{d t} \delta q=C_{V} \delta V+C_{\alpha} \delta \alpha+C_{q} \delta q+C_{\alpha} \delta \dot{\alpha}+C_{\delta e} \delta e \\
& \frac{d}{d t} \delta \theta=\delta q
\end{aligned}
$$


where the values of the stability derivatives can be found in most college level textbooks in the field, e.g. R ef. [9]. In many cases the stability derivatives can be assumed to be constant over time interval comparable to the period of the slow varying parameters so system (2) becomes a linear system with constant coefficients.

The standard assumption of $\delta V=0$ over short time intervals further yields a second order equation that describes the variation of angle's of attack perturbations; this equation that can be written as a standard second order ODE,

$$
\begin{aligned}
& (\delta \ddot{\alpha})+2 \zeta_{1}(\delta \dot{\alpha})+p_{1}{ }^{2}(\delta \alpha)= \\
& \quad=\left(-B_{\gamma}^{2}+C_{q} B_{\gamma}\right)(\delta \gamma)+\left(C_{\delta e}+C_{q} B_{\delta e}-B_{\gamma} B_{\delta e}\right)(\delta e)-B_{\delta e}(\delta \ddot{e})
\end{aligned}
$$

where

$$
\zeta_{1}=\left(B_{\alpha}-C_{q}-C_{\dot{\alpha}}\right) / 2 \text { and } p_{1}^{2}=B_{\gamma} B_{\alpha}-C_{\alpha}-B_{\alpha} C_{q}
$$

and for stability conditions $\zeta_{1}^{2}<p_{1}^{2}$.

A nalytical solutions discussed herein only address the cases of systems for which the fast modes are stable. Analyses of systems with unstable fast modes exceed the limits of this paper; such are useless without automatic stabilization systems, so the complexity of the model s increase significantly.

For stable systems, equation (3) has analytical solution, which varies with the nature of control that is applied, here with the elevator control. For the case of a constant elevator deflection, assuming that the perturbation in slope will remain constant over a short time frame, the perturbation of the angle of attack may be written as

$$
(\delta \alpha)=A_{1} e^{-\zeta_{1} t} \sin \left(\sqrt{\Omega_{1}} \cdot t+v_{1}\right)+\frac{\left(-B_{\gamma}^{2}+C_{q} B_{\gamma}\right)(\delta \gamma)+\left(C_{\delta e}+C_{q} B_{\delta e}-B_{\gamma} B_{\delta e}\right)(\delta e)}{p_{1}^{2}}
$$

where $\Omega_{1}=p_{1}^{2}-\zeta_{1}^{2}$ and the integration constants are written function of the initial conditions, for example $t=0, \delta \alpha=(\delta \alpha)_{0}, \delta \dot{\alpha}=(\delta \dot{\alpha})_{0} \cong(\delta q)_{0}$ and the particular solution $(\delta \alpha)_{P}$ as

$$
A_{1}=\frac{(\delta \alpha)_{0}-(\delta \alpha)_{p}}{\sin v_{1}} \text { and } \tan v_{1}=\frac{\left[(\delta \alpha)_{0}-(\delta \alpha)_{p}\right] \sqrt{\Omega_{1}}}{(\delta q)_{0}+\zeta_{1}\left[(\delta \alpha)_{0}-(\delta \alpha)_{p}\right]}
$$

and the particular solution is the second term on the right hand side of eq.(5)

\subsection{The angle of attack reduced model}

Neglecting smaller terms in eq.(1), M oraru [10] wrote the equations of motion as 


$$
\begin{aligned}
& \dot{V}=-\frac{\rho S C_{D}}{2 m} V^{2}-g \sin \gamma+\frac{T}{m} \\
& \dot{\gamma}=-\frac{g \cos \gamma}{V}+\frac{\rho S C_{L \alpha}}{2 m} V \alpha \\
& \ddot{\theta}=\frac{\rho S c}{2 J} V^{2} C_{m \alpha} \alpha+\frac{\rho S c}{2 J} V^{2} C_{m q} q+\frac{\rho S c}{2 J} V^{2} C_{m \delta e} \delta e
\end{aligned}
$$

Next, for systems whose inertia properties and thrust effect $\left(a_{T}=T / m\right)$ can be considered constant over short intervals (comparable to the period of the fast variables) the parameters [10]

$$
K_{D}=\frac{\rho S C_{D}}{2 m} ; K_{L}=\frac{\rho S C_{L}}{2 m} ; K_{M}=\frac{\rho S c C_{m \alpha}}{2 J_{y}} ; K_{q}=\frac{\rho S c \tilde{C}_{m q}}{2 J_{y}} ; K_{\delta e}=\frac{\rho S c C_{m \delta e}}{2 J_{y}}
$$

vary very little for short time intervals, so, with velocity and the slope almost constant. (i.e. employing the usual assumption utilized to differentiate the fast and slow modes), the angle of attack may be described by a constant coefficients equation

$$
\ddot{\alpha}+2 \zeta_{2} \dot{\alpha}+p_{2}^{2} \alpha=g \cos \gamma\left[K_{D}-c K_{q}+\frac{2 g \sin \gamma}{V^{2}}+\frac{K_{\delta e} V^{2} \delta e}{g \cos \gamma}\right]
$$

where

$$
\begin{gathered}
\zeta_{2}=\left(K_{L}-c K_{q}\right) V / 2 \\
p_{2}^{2}=-K_{M} V^{2}-K_{L} K_{q} c V^{2}-K_{L} K_{D} V^{2}+K_{L} a_{T}
\end{gathered}
$$

and, again, stability considerations require $p_{2}^{2}>\zeta_{2}^{2}$

M oraru [5,6], Safta [11] and M oraru and Safta [12] also developed even more general equations for the angle of attack, however, this will not be discussed here, as the main purpose of this paper is to address cases of simple solutions with easy to obtain and implement analytical solutions. As mentioned before, significantly more elaborate analytical models exists in literature, analytical solutions were obtained for some cases, however, nowadays analytical solutions that spread over several pages, although of great interest in the previous decades, are no longer utilized and have been replaced by purely numerical methods.

For stable systems, equation (9) has analytical solutions, which, again, vary with the nature of control that is applied, here with the elevator deflection. For the case of a constant elevator deflection, assuming that the slope remains constant over a short time frame, the angle of attack varies in time as

$$
\alpha=A_{2} e^{-\zeta_{2} t} \sin \left(\sqrt{\Omega_{2}} \cdot t+v_{2}\right)+\frac{g \cos \gamma\left[K_{D}-c K_{q}+\frac{2 g \sin \gamma}{V^{2}}+\frac{K_{\delta e} V^{2} \delta e}{g \cos \gamma}\right]}{p_{2}^{2}}
$$


where, again, $\Omega_{2}=p_{2}^{2}-\zeta_{2}^{2}$ and, same as before, the integration constants $A_{2}$ and $v_{2}$ may be written function of the initial conditions, $\alpha(0)=\alpha_{0}, \dot{\alpha}(0)=\dot{\alpha}_{0} \cong q_{0}$ and the particular solution $\alpha_{P}$ as

$$
A_{2}=\frac{\alpha_{0}-\alpha_{p}}{\sin v_{2}} \text { and } \tan v_{2}=\frac{\left(\alpha_{0}-\alpha_{P}\right) \sqrt{\Omega_{2}}}{q_{0}+\zeta_{2}\left(\alpha_{0}-\alpha_{P}\right)}
$$

and the particular solution is the second term on the right hand side of eq.(12)

\section{Results and discussion}

The validity of the analytical solutions presented above may vary from case to case and it should be checked for actual conditions. However the errors over short time intervals, comparable to the period of the fast variables, may be expected to remain small, of the order of a few percentages.

Just for illustrative purposes, some numerical examples are presented below, which are based upon a hypothetical aircraft whose aerodynamic coefficients are given in Table 1. The reference surface of the wing is $13.18 \mathrm{~m}^{2}$ and the aerodynamic chord is $2.45 \mathrm{~m}$. The mass of the airplane is $9874 \mathrm{~kg}, J_{y}=66591 \mathrm{~kg} \mathrm{~m}^{2}$ and it is assumed that during the maneuver they remain constant.

Figure 1 presents the responses to a $3^{0}$ step function televator deflection, applied to the airplane assumed in steady level flight with speeds of 150,300 and $450 \mathrm{~m} / \mathrm{s}$, respectively, at an attitude of $1000 \mathrm{~m}$. Data provided by a $4^{\text {th }}$ order Runge K utta numerical integration of eq. (1) are compared against data obtained equations (5) and (12). The agreement is good, the errors remain within a few percentages.

Table.1 Aerodynamic Data

\begin{tabular}{|c|c|c|c|c|c|c|c|c|c|}
\hline $\mathrm{M}$ & $\mathrm{C}_{\mathrm{x} 0}$ & $C_{D}^{\alpha^{2}}$ & $C_{D}^{\beta^{2}}$ & $\mathrm{C}_{\mathrm{L}}{ }^{\alpha}$ & $\mathrm{C}_{\mathrm{L}}{ }^{\beta}$ & $\mathrm{C}_{\mathrm{m}}{ }^{\alpha}$ & $\mathrm{C}_{\mathrm{m}}{ }^{\beta}$ & $C_{m \alpha}^{*}$ & $C_{m q}$ \\
\hline 0.4 & 0.0267 & 5.032 & 0.984 & 8.032 & 1.979 & -1.982 & -3.447 & -1.98 & -0.27 \\
\hline 0.6 & 0.0268 & 5.344 & 1.026 & 8.344 & 2.063 & -2.158 & -3.594 & -2.16 & -0.14 \\
\hline 0.8 & 0.0269 & 5.866 & 1.116 & 8.866 & 2.233 & -2.408 & -3.892 & -2.41 & -0.095 \\
\hline 0.9 & 0.0302 & 6.315 & 1.211 & 9.352 & 2.415 & -2.725 & -4.241 & -2.61 & -0.079 \\
\hline 1.0 & 0.0444 & 7.742 & 1.374 & 10.74 & 2.747 & -3.105 & -4.808 & -2.95 & -0.072 \\
\hline 1.1 & 0.0736 & 8.551 & 1.457 & 11.55 & 2.915 & -3.355 & -5.114 & -3.23 & -0.069 \\
\hline 1.2 & 0.0736 & 7.825 & 1.374 & 10.83 & 2.747 & -3.548 & -4.974 & -3.32 & -0.067 \\
\hline 1.3 & 0.0714 & 7.083 & 1.261 & 10.18 & 2.486 & -3.627 & -4.686 & -3.35 & -0.064 \\
\hline 1.4 & 0.0694 & 6.347 & 1.142 & 9.347 & 2.273 & -3.483 & -4.205 & -3.25 & -0.058 \\
\hline 1.6 & 0.0639 & 4.571 & 0.901 & 7.571 & 1.748 & -2.525 & -3.263 & -2.85 & -0.049 \\
\hline 2 & 0.0473 & 2.621 & 0.653 & 5.621 & 1.208 & -1.765 & -2.465 & -2.62 & -0.034 \\
\hline
\end{tabular}




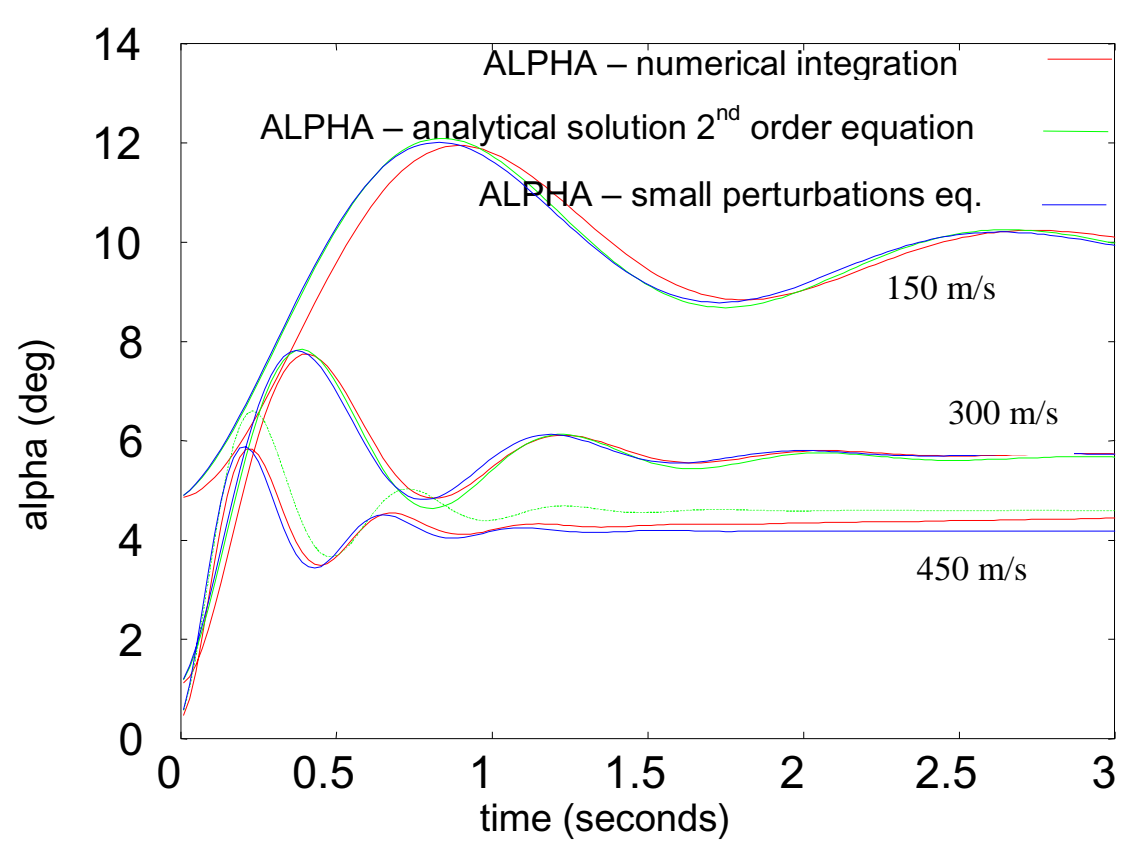

Fig. 1. A ngle of attack as provided by the three models

\section{Conclusion}

Although they have been often phased out by purely numerical techniques, the analytical solutions in flight dynamics may still be of interest, as they can be successfully utilized, eventually integrated within modern numerical algorithms to accelerate them. The current paper presents the main equations of two analytical models for the angle of attack. He first analytical solution that is presented was obtained for an equation written in terms of perturbations, while the second model include an equation obtained from a simplified set of equations of motion (neglecting the small contributions). For time-intervals comparable to the period of the small variables, the results provided by the analytical solutions agree well with the numerical results obtained via a $4^{\text {th }}$ order R unge K utta numerical integration of the equations of motion.

The work has been co-funded by the Sectoral Operational Programme Human Resources Development 2007-2013 of the Romanian M inistry of Labour, Family and Social Protection through the Financial A greement POSDRU/89/1.5/S/62557

\section{References}

1. J.B. Rosser, R.R.Newton, L.G.Gross, Mathematical Theory of Rocket Flight, M cGrawHill, New York (1947)

2. H. M olitz, R. Strobel, Aussere Ballistik, Springer V erlag, (1963) 
3. M. Niţă, D.Şt. Andreescu, The Flight of the rocket, The Military Publishing House, Bucharest, (1964) (in Romanian)

4. F. Moraru, Studies and researches of applied mechanics (in Romanian), number 4, vol.31, pp. 753-779, The Romanian A cademy, 1972

5. F. M oraru, PhD. Dissertation, The Polytechnic Institute of Bucharest, R omania (1972)

6. M oraru, F., The Bulletin of the Military A cademy, N o.1, Bucharest, Romania (1973)

7. N. A nanthkrishnan and S. Unnikrishnan, J. of Guidance, Control and Dynamics, 24, No.6, (2001)

8. N. Ananthkrishnan, AIAA 2004-4950, AIAA Atmospheric Flight Mechanics Conference and Exhibit, Providence, Rhode Island, (2004)

9. B. Etkin, Dynamics of Atmospheric Flight, W iley, ISB N : 978-0-471-24620-6 (1972)

10. F. M oraru, Conference in A coustics, Book 24, The Romanian A cademy, B ucharest, (October 1994).

11. D. Safta, PhD. Dissertation, Technical M ilitary A cademy, B ucharest, R omania (1996)

12. F. M oraru, Safta, D., $2^{\text {nd }}$ Conference of B arrel Weapon Systems, M ilitary A cademy in B rno, Czech Republic (1997) 